\title{
Cultural and Educational Impact of New Formation Perspectives of Parashqevi Qiriazi
}

\author{
PhD. Rregjina Gokaj \\ University "Aleksander Moisiu", Durres Albania \\ Email: gokajregji@yahoo.it \\ PhD. Esmeralda Hoti \\ University "Aleksander Moisiu", Durres Albania \\ Email: esmeraldahoti@yahoo.it
}

\section{Doi:10.5901/mjss.2014.v5n16p712}

\section{Abstract}

\begin{abstract}
The realization of teaching programmes became one of the most important privileges during the Albanian National Movement. One of the major representatives who elaborated a new teaching programme of the Albanian educational system during the first decade of the twentieth century, based on the deepened analysis of the ottoman and United States teaching system, it was the teacher and the director of the school for Little Girls, Parashqevi Qiriazi. In her new proposals, Qirjazi included various pedagogical theories relevant to the European context denoting a contemporaneous scientific preparation. In order to realize the patriotic education, the Albanian woman, like the Albanian patriots have so far sustained in general on their books and articles, their own civilization should come first, that is teaching implied. It was difficult to avoid the situation of the Albanian women at the time, which also was pending on the social developments within the insiders of the ottoman society.
\end{abstract}

La realizzazione dei programmi d'insegnamento diventa una delle prerogative principali del Movimento nazionale albanese, e uno dei maggiori rappresentanti che elabora un nuovo programma educativo del sistema d'istruzione albanese durante il primo decennio del XX secolo, basandosi sull'analisi approfondita del sistema d'istruzione ottomano e statunitense, fu la maestra e la direttrice della scuola per fanciulle Parashqevi Qiriazi (Osmani, 1998, p. 173). II suo programma, intitolato Lo sviluppo delle scuole turche e il sistema ideale dell'istruzione in Albania, fu presentata per la prima volta al governo temporaneo di Valona nel 1913, mentre fu resa nota solo nel 1919 grazie alla pubblicazione del libro di Kristo Dako - il nipote di Pareshqevi Qiriazi - Albania, the Master Key to the Near East e i materiali esposti nella rivista La stella del Mattino (Yll' i Mëngjesit) (Osmani, 1997, pp. 129-147).

Nelle nuove proposte educative di Qiriazi si includevano diverse teorie pedagogiche rilevanti del contesto europeo dimostrando una contemporanea preparazione scientifica. Così, durante il periodo d'asilo, inclusi gli asili per gli orfani (Osmani, 1997, pp.129-147), gli alunni dovevano apprendere conoscenze sull'ambiente, sulle espressioni morali e sulle capacità di lettura e scrittura, per lo sviluppo del quale, come suggeriva la Montessori (Moletto, Zucchi, 2003, p.16) «gli insegnanti devono osservare come i bambini imparino dalle madri a pronunciare le prime parole e poi le prime frasi, questo senza che queste madri abbiano mai letto un libro di didattica e di pedagogia».

\section{Le fasi della donna Albanese}

Per realizzare l'educazione patriottica, la donna albanese, come da diversi anni i patrioti albanesi avevano ampiamente sostenuto nei loro libri e articoli, in primo luogo doveva impegnarsi nella propria emancipazione che, dunque, implicava l'istruzione. Inevitabilmente la situazione delle donne albanesi dipendeva anche dagli sviluppi sociali all'interno della società ottomano. In realtà soltanto dopo la nuova situazione politica generata dalla proclamazione della costituzione, si apriva una spirale che offriva nuove opportunità alle donne, in particolare di religione musulmana, come riferimento alle proprie aspirazioni politiche e sociali, insieme al loro desiderio di istruirsi. Ricordiamo inoltre che solo nel 1911 fu costruito il primo liceo per ragazze (Dumont, 2011, p.630).

È in questo clima che s'inserisce l'attività di P. Qiriazi e della sorella Sevasti. Queste grandi donne fondarono la prima associazione tutto al femminile, chiamata La Stella del Mattino ${ }^{1}$ appena rientrate a Korça, il 29 gennaio del 1909,

${ }^{1} \mathrm{Nel}$ Regolamento (Kanonizma) dell'Associazione pubblicato nel 1912, incontriamo tutta una seria di regole a scopo organizzativo e
educativo. Cosi citiamo: «1. A Korça le signore hanno fondato un'associazione intitolata La Stella del Mattino; 2. Lo scopo 
dopo la partecipazione nel congresso di Manastir. L'associazione era aperta a tutte le giovani donne provenienti da svariati strati sociali e diverse religioni come l'unica via verso la costruzione di un accogliente ambiente famigliare e nazionale. Obiettivo principale fu quello di istruire le giovani donne a essere delle buone madri e delle degne cittadine, poiché per Parashqevi il dovere da intellettuali era di «preparare le ragazze, addestrarle a essere autosufficienti, coltivare i loro potenziali fisici, morali ed estetici, per riuscire a dare una buona educazione ai propri figli» ("Korça", 1912, pp. 3-15).

Riuscì con fermezza nell'intento di presentare a tutto lo spettro intellettuale albanese la propria visione sullo sviluppo dell'istruzione infantile, suggerendo agli insegnanti, per esempio, la presenza dei giochi e degli addestramenti fisici durante l'interazione con i bambini per un appropriato sviluppo psicologico. Attraverso quest'ottica si potrebbe osservare che per realizzare e applicare i programmi d'istruzione nel delicato contesto culturale ed educativo albanese bisognava prendere in considerazione l'approccio teorico montessoriano, secondo il quale, ricordiamo, «i bambini godono di molto spazio, imparano giocando, suddividono autonomamente la giornata, lavorano molto anche con le mani [...], in più oltre alle normali materie di studio, i bambini imparano ad occuparsi di cose pratiche, sviluppando la propria creatività, acquisiscono fiducia in se stessi e imparano a conoscersi» (Giovetti, 2007, p. 56).In quest'ottica, presentando il suo progetto educativo, P. Qiriazi si allaccia al pensiero di Rousseau, secondo il quale «l'autentica educazione comporta la consapevolezza che essa non è un atto puramente intellettuale, ma il risultato della concreta e diretta esperienza unita alla riflessione personale [...]. È necessario che l'educatore rispetti la personalità del fanciullo in tutta la sua integrità e nella gradualità del suo sviluppo. Egli deve tanto formare positivamente questa personalità, ma consentirle e favorirne il libero sviluppo» (Favorini, 2012, p. 87).

In secondo luogo, per quando riguardava le scuole elementari, estese l'età a sei anni e le suddivise in due cicli da tre anni ciascuno, condividendo l'idea secondo la quale il bambino dovesse cominciare la prima elementare a sei anni (Dishnica,1997, pp. 89).Queste scuole dovevano avere come obiettivo pedagogico la formazione della personalità del cittadino albanese tramite l'apprendimento della scrittura, della lettura, dell'aritmetica, della storia, della geografia, del disegno, della confidenza con l'ambiente e la musica locale (Osmani,1998, p.177). I ragazzi e le ragazze negli asili e nelle scuole elementari potevano svolgere le lezioni insieme, mentre durante la scuola media separatamente, per frequentarsi da tutti ( Dishnica,1997, pp. 90).

In terzo luogo, le scuole secondarie, suddivise in due cicli di quattro anni, proponevano la «formazione di un degno cittadino, intelligente e con la mente aperta», capace di orientarsi con consapevolezza verso professioni utili e importanti. L'età media degli alunni di queste scuole doveva essere di tredici anni. I metodi pedagogici e psicologici in queste scuole dovevano adattarsi allo sviluppo cognitivo del bambino. Nei primi quattro anni venivano insegnate le materie di Lingua, Geometria, Aritmetica, Lettura, Lingua straniera (francese, inglese, tedesco), Geografia, Musica, Botanica, Zoologia, Fisica, mentre durante il secondo quadriennio si prevedevano queste le materie di Lingua classica, Lingua straniera, Chimica, Storia, Trigonometria, Fisiologia, Educazione Fisica e Manodopera (Dishnica,1997, pp. 91).

In quarto luogo, l'Università, secondo la proposta di P. Qiriazi, doveva durare quattro o cinque anni a seconda delle facoltà, prescelte secondo le necessità di sviluppo del paese. Nelle università albanesi le lezioni dovevano essere comuni sia per ragazzi sia per ragazze, basandosi sulla migliore esperienza contemporanea (Dishnica, 1997, pp. 92).

Alla fine, le scuole per bambini disabili erano viste come un mezzo utilissimo per la loro riabilitazione, in particolar modo dei sordo-muti, mentre per quando riguarda le scuole private, P. Qiriazi era dell'idea di sottoporle alla stretta sorveglianza della Stato (Dishnica,1997, pp. 92).

\section{Organograma}

Come un'acuta osservatrice delle teorie di Dewey e Montessori, P. Qiriazi modellò il suo laborioso progetto di sviluppo dell'istruzione dal punto di vista amministrativo-statale e organizzativo-pedagogico prendendo in considerazione innanzitutto le attitudini psicologiche del popolo albanese, le necessità più emergenti educative del tempo e la costruzione dell'infrastruttura di insegnamento di matrice europea (Osmani,1998, p. 175) :

dell'associazione è di divulgare l'istruzione tra le signore albanesi e per trattenere le ragazze povere che studiano; 3. L'associazione avrà una presidentessa, una vicepresidentessa, una bibliotecaria, una cassiera e una segretaria; 4. II Consiglio sarà costituito da sette signore scelte da una votazione generale; 5. L'associazione svolgerà delle lezioni gratis; 6. I membri non possono avere meno di 20 anni; 7. L'Associazione avrà anche un membro d'onore scelto dal Consiglio; 8. Ogni membro pagherà 1 grosh al mese e non più di 24 lira all'anno; 9. II Consiglio farà il bilancio annuale in una riunione generale; 10. La cassiera non può eseguire nessun pagamento senza il permesso del Consiglio; 11. L'associazione avrà un proprio timbro che lo terrà solo la presidentessa; [...] 15. L'associazione non ha scopi politici; [...] 19. II Governo ha il pieno diritto di ordinare la chiusura dell'associazione per qualsiasi motivo» ("Korça", 1912, pp. 315). 


\section{Parlamento $\leftarrow$ Ministero d'Istruzione $\leftarrow$ II Consiglio istruttivo della città $\leftarrow$ Scuole $\uparrow$ Università}

Gli asili (4-6 anni) - Le scuole elementari (6-12 anni) - Le scuole secondarie (12/13-20 anni) - Le scuole universitarie (20-23 anni) - Le scuole per gli handicap - Le scuole private

«Lo scopo primo e vero dell'istruzione», affermava l'autrice, «non è di darvi conoscenze straordinarie, ma di farvi coltivare le risorse interiori e di costruirvi valori universali. In sintesi, lo scopo dell'istruzione è di prepararvi a vivere con dignità e utilità la nostra vita» (Osmani,1997, pp.149-152). Atteggiamenti che ci fanno ricordare il pensiero pedagogico di Pestolazzi, che influenzò notevolmente la sua progettazione educativa, il quale sosteneva che «l'educazione deve essere vissuta come universale (nel senso di piena socialità) e concreta (nei confronti della propria esistenza e nelle relazioni con gli altri). [...] Ordine della natura nell'educazione dell'umanità è la forza dell'applicazione e dell'esercizio delle sue cognizioni, dei suoi doni, delle sue capacità» (Stroppa,2001, p. 137).

Nella sua proposta pedagogica Parashqevi non esita di descrivere nei dettagli quali sarebbero stati gli avantaggi psicologici e sociali di una buona educazione scolastica. Così, «la storia della pedagogia ci insegna, dopo il nuovo approccio teorico di Lutero, che l'istruzione non ha come obiettivo ultimo insegnare dei semplici fatti della vita, ma sviluppare intrinseche attitudini capaci di farli propri questi fatti. [...] Con semplici parole l'istruzione è applicazione e [...] l'insegnante deve ricordare di trovare sempre un associazione tra l'ambiente sociale e quello scolastico» (Osmani,1997, pp. 153-154).

Voleva, fondamentalmente, giovani capaci di riflettere sul proprio pensiero e sulle caratteristiche culturali albanesi, cioè non limitarsi a ripeterne il pensiero altrui, poiché, come Benedetto Croce, grande filosofo italiano, ci fa capire, rischiano di trasformarsi in cattivi discepoli che «scimmiottano il loro maestro e ne ripetono le idee e i pensieri, alcuni con una negatività, cioè attraverso un rifiuto ribelle; mentre i buoni discepoli si distinguono dai cattivi per il fatto di riprendere i problemi del maestro in una nuova luce, di considerarli da un'altra prospettiva e di dedicarsi, soprattutto, alle domande alle quali egli non è stato capace di rispondere» (Böhm,2000, p. 71).

Una buona educazione richiede di fermarsi e di osservare la natura del bambino, di rivelarvi i suoi desideri e collaborare per realizzarli, considerando che l'istruzione non deve essere data per scontato ma deve essere considerato un forte stimolo verso la proiezione delle proprie attitudini cognitive ed emotive di tutti, giovani e non, ricchi e poveri, musulmani 0 cristiani, commercianti o semplici lavoratori, tutti, dopo aver sviluppato certe capacità riflettive, «hanno il diritto di aprire le porte della conoscenza», una conoscenza che, per Parashqevi, doveva cominciare dalla storia dell'Albania. Ogni cittadino di questo paese doveva familiarizzarsi con certi concetti e testimonianze del proprio passato come l'unica via per riconoscersi e identificarsi e i libri che potevano sostenere la sua tesi erano: Chi sono gli albanesi (Cilët janë Shqipëtaret) di Z. CH. Dako, L'Albania che cos'è stata, che cos'è e che cosa sarà (Shqipëria ç'ka qënë, ç'është dhe çdo të jete), Storia generale (Historia e përgjithshme), di Sami Frashëri e Storia di Skënder Beu (Histori e Skënder Beut) di Naim Frashëri. I giovani non dovevano studiare solo la loro storia nazionale, ma anche il passato delle altre nazioni per prevenire certi avvenimenti e osservare il proprio grado di progresso (Böhm, 2000, p. 71).

\section{Conclusioni}

Nell'analisi fin qui presentata possiamo soffermarci su un aspetto che affiora dai nuovi approcci teorici pedagogici proposti, ossia il rapporto educazione-società, qualche richiama il concetto della pedagogia sociale, emerso nel corso del XIX secolo grazie al lavoro di Natorp² (Graminia, 2003, p. 27). Possiamo sostenere che la fisionomia della nuova pedagogia albanese stava per compiere una metamorfosi teorica grazie anche agli avvenimenti politici e culturali, permettendo di rispettare le condizioni di una vera e propria pedagogia sociale secondo le visioni proposte da Gracia Garrido, nel 1971, e che considerano la pedagogia sociale in funzione dello spazio dove si sviluppa la società, come la trasmissione dei valori educativi propri di una determinata società, come influenza educativa che la società nel suo insieme esercita sul cittadino e come influenza dei poteri pubblici con fini politici o per la vita sociale (Graminia, 2003, p. 27).

In sintesi, l'ideale istruttivo proposto da Parashqevi Qiriazi può essere espresso con una sola frase: «quello che lo scultore fa alla pietra, l'istruzione lo fa all'anima dell'essere umano» (Graminia, 2003, p. 27).

\footnotetext{
2 Paul Natorp (1854-1924) fu un filosofo tedesco rappresentante della scuola di Marburgo, fautrice di un ritorno al criticismo. (Enciclopedia Universale, 2006, p. 1122)
} 


\section{Reference}

Böhm W., Storia della pedagogia. Da Platone ai nostri giorni, Armando Editore, Roma, 2007.

Dishnica, Motrat Qiriazi, Enciklopedike, Tiranë, 1997.

Dumont, Georgeon, La morte di un impero (1908-1923), In Mantran R. (a cura di), Storia dell'impero ottomano, ARGO, Lecce, 2011.

Enciclopedia Universale, Corriera della Sera, Milano, 2006.

Favorini, A. M. (a cura di), Spiritualità, benessere e qualità di vita, Franco Angeli, Milano, 2012.

Giovetti P., Indaco. Bambini realtà del terzo millennio, Edizione Mediterranee, Roma, 2007.

Graminia A., Manuale di pedagogia sociale: scenari del presente e azione educativa, Armando Editore, Roma, 2003.

Kanonizama e Shoqërise zonjavet "YIl' i Mëngjesit", In "Korça", Korçë, 1912.

Moletto A., Zucchi R., La metodologia pedagogica dei genitori, Maggioli Editore, Firenze, 2003.

Osmani Sh., Reflekse etnopedagogjike, "Idromeno", Tiranë, 1998.

Osmani Sh., Trashëgimi social-pedagogjike, Motrat Qiriazi, Grafikprint, Prishtinë, 1997.

Stroppa C., Jan Amos Comenius e il sogno urbano, Franco Angelo, Milano, 2001. 